\title{
High Performance and Efficient Fluid Power Actuation: Challenges in Control and Diagnosis and Design
}

\author{
Nariman Sepehri \\ Department of Mechanical Engineering, Faculty of Engineering \\ University of Manitoba \\ Winnipeg, Manitoba, Canada
}

\begin{abstract}
When it comes to properties such as combining power and mobility, easy realization of linear motion, ability to perform in extreme environments and flexibility in packaging, there is arguably no other technology that can compete with hydraulic fluid power. For this reason, fluid power systems are used in many important applications: aviation (flight control actuators), automotive (active suspension), manufacturing (rolling mills), training (motion simulators), off-highway (earth-moving machines) and robotics (underwater hydraulic manipulators). In this talk, we will describe research conducted to improve reliability of hydraulically-actuated systems, by understanding failure mechanisms and designing fault diagnosis and fault tolerant controls for hydraulic machines. Other projects that will be described are development of control concepts for hydraulic/pneumatic manipulators targeting environmental interaction or cooperative tasks, and understanding energy efficiency in mobile hydraulic equipment.
\end{abstract}

Keywords: Fluid power systems, hydraulic and pneumatic actuators, control synthesis, teleoperation and robotics, energy efficiency. 